\title{
Preventive Cancer Screening in Asian Americans: Need for Community Outreach Programs
}

\author{
Gregory Wu*, Niki T Augustine, Steve Sung Kwon*
}

\begin{abstract}
Background: Asian Americans (AA) are the only racial group in the United States to experience cancer as the number one cause of mortality. Yet, Asian Americans have one of the lowest rates of cancer screenings of all minority groups in the United States. Methods: A cross-sectional and population-based study design was used. Cross-sectional data was collected from 1,650 AA participants via a survey given during two annual community health festivals in 2017 and 2018. Survey variables included sociodemographic measures, self-reported English-language proficiency level, access to primary care, attitudes on preventative cancer screening, current screening status and barriers to undergoing cancer screening. Results: Nearly $66 \%(\mathrm{n}=1,081)$ reported not having a primary care physician $(\mathrm{PCP})$. While the majority of the participants $(\mathrm{n}=1,510,92 \%)$ stated that preventative cancer screenings were important, only a small portion ( $\mathrm{n}=1,091,16 \%)$ were up-to-date on cancer screening procedures. The biggest barriers to preventative cancer screening were: 1) Lack of insurance ( $\mathrm{n}=840,40 \%) ; 2$ ) Cost of seeing a physician $(\mathrm{n}=517,24 \%)$; and 3 ) Do not feel the need ( $n=299,14 \%)$. Conclusions: To overcome the barriers we identified and effectively increase cancer screenings in Asian Americans, community outreach should be considered to provide linkage to primary care physicians and navigation to low-cost screening programs.
\end{abstract}

Keywords: Healthcare disparities- cancer- Asian Americans- screening- community outreach

Asian Pac J Cancer Prev, 23 (2), 393- 397

\section{Introduction}

Cancer is the leading cause of death in Asian Americans (AA) (Hastings et al., 2015; Torre et al., 2016; CDC, 2019). AAs are the only racial group in the United States to experience cancer as the number one cause of mortality, accounting for $27 \%$ of all deaths (American Cancer Society, 2016). Despite the identification of best practices for early detection through screenings, AAs have one of the lowest rates of preventive cancer screenings of all minority groups in the United States (Chen, 2005; Bodle et al., 2008; Hung et al., 2014; Torre et al., 2016; Ratushnyak et al., 2019; Jun, 2020). Barriers to cancer screenings continue to exist among Asian Americans, which include a lack of patient navigation, cultural and linguistic unfamiliarity, lack of insurance, fear of testing, cost, lack of awareness of the disease, lack of prevention and treatment, lack of time, and lack of access to healthcare providers (Ma et al., 2009; Ma et al., 2009).

The purpose of this study is to understand the attitudes, identify barriers, and address disparities in cancer screening among AAs in Northern New Jersey. The goal is to promote a preventive care mindset to cancer in AAs through community outreach that will lead to earlier detection and ultimately improved cancer-specific mortality. Identification of documented barriers may lead to actionable recommendations for policymakers, healthcare providers, community advocates, and hospital system partners working within these populations to address and limit disparities in cancer screening among AAs.

\section{Materials and Methods}

Design

A cross-sectional study design was used to determine the gaps in healthcare and preventive care amongst the AA community. A 12-question survey was employed to identify perceptions of cancer screenings specific to AAs. The survey assesses sociodemographic measures, self-reported English-language proficiency level, access to primary care, attitudes on preventative cancer screening, current screening status, and barriers to undergoing cancer screening. The survey distributed was anonymous and our data collection and analyses did not contain any identifiers.

\section{Study Sample}

The Asian Health Services (AHS), established at Holy Name Medical Center, was developed to address gaps in language and provide culturally appropriate healthcare

Department of Surgery, Holy Name Medical Center, Teaneck, NJ, USA. *For Correspondence: gregorywu94@gmail.com,skwon@holyname.org 
services for the AA population in the greater New York and New Jersey area. One of its outreach programs is the Annual Community Health Festival, which attracts more than 1,200 AAs from the tri-state area during September. During this festival, volunteer physicians provide services (blood tests, consults, exams/screenings, vaccines, EKGs) to mostly uninsured or underinsured AAs.

AAs who attended the festival in 2017 and 2018 were recruited to participate in the survey. Between the two festival dates we had a total of 1,835 attendees, there were 1,023 attendees from 2017 and 812 from 2018 that were offered the survey. A total of 1,650 Asian Americans completed the survey and were included in the study. There were no duplicate surveys from a single participant for both years. The survey was offered in English, Chinese, Japanese or Korean.

\section{Measures}

Each survey consisted of 12 questions that assessed demographic characteristics, primary care provider access, current status of own cancer screenings, perceived importance of preventive care, knowledge of cancer screening procedures, and barriers to cancer screening. Demographic characteristics included age, gender, annual income level, and education level. For patients who checked multiple boxes for education level, only the highest value as included in the analysis.

To begin the assessment, we first asked participants "Do you have a primary care physician?" and "How regularly do you see your primary physician?" as a measure for patients access to primary care. Respondents were then asked whether they were up-to-date on cancer screening procedures. To assess attitudes on preventive care and cancer screenings, participants were asked how important preventive care was to them on a scale of "Not Important' to "Very Important." Respondents were also asked if they knew what cancer screenings were needed for their age. To assess for barriers in preventive care, participants were instructed to select all the reasons for not undergoing cancer screening procedures and seeing a primary on a regular basis. ("Lack of Insurance," "Cost of Visit," "Do not feel need to see PCP regularly," "Language Barrier," "Fear," "Lack of Transportation"). Those who failed to answer select questions were marked as "N/A."

\section{Analysis}

The distribution of each category was reviewed from the collected survey answers. Percentiles were calculated to summarize variables (age, sex, insurance status and type, income levels, and education levels). STATA was used for all cross-sectional analyses (Version 16; STATACorp, College Station, TX).

\section{Results}

\section{Description of the Sample}

The sample consisted of 1,650 participants from the two festival dates in Fall 2017 and 2018. We had a total of 1,835 participants register to attend with a sign-up sheet, suggesting a response rate of around 90\%. Festival attendees came from as far south as South Jersey and north as Massachusetts (Figure 1). The majority of the participants were female $(n=1,004,61 \%)$ and $38 \%$ $(n=642)$ were male. The largest age group in our sample

Table 1. Demographic, Screening Status and Attitudes of Survey Respondents

\begin{tabular}{|c|c|}
\hline Characteristic & All Patients Screened $n=1650(\%)$ \\
\hline \multicolumn{2}{|l|}{ Age } \\
\hline$<20$ & $13(0.78)$ \\
\hline $20-30$ & $73(4.24)$ \\
\hline $31-40$ & $137(8.30)$ \\
\hline $41-50$ & $296(17.94)$ \\
\hline $51-60$ & $533(32.3)$ \\
\hline $61-70$ & $399(24.18)$ \\
\hline$>70$ & $166(10.06)$ \\
\hline N/A & $33(2)$ \\
\hline \multicolumn{2}{|l|}{ Gender } \\
\hline Male & $642(38.91)$ \\
\hline Female & $1004(60.85)$ \\
\hline N/A & $4(0.24)$ \\
\hline \multicolumn{2}{|l|}{ Income } \\
\hline$<\$ 25,000$ & $696(42.18)$ \\
\hline$\$ 25,000-\$ 49,999$ & $506(30.67)$ \\
\hline$\$ 50,000-\$ 74,999$ & $231(14)$ \\
\hline$\$ 75,000-\$ 149,000$ & $91(5.52)$ \\
\hline$>\$ 150,000$ & $22(1.33)$ \\
\hline N/A & $104(6.30)$ \\
\hline \multicolumn{2}{|l|}{ Education Level } \\
\hline No Formal Education & $33(2)$ \\
\hline High School/Equivalent & $436(26.42)$ \\
\hline Some College & $299(18.12)$ \\
\hline Associate Degree & $40(2.42)$ \\
\hline Bachelor's Degree & $607(36.78)$ \\
\hline Master's Degree & $149(9.03)$ \\
\hline Professional Degree & $23(1.39)$ \\
\hline Doctorate Degree & $24(1.46)$ \\
\hline N/A & $39(2.36)$ \\
\hline \multicolumn{2}{|c|}{ Have A Primary Care Provider (PCP) } \\
\hline Yes & $431(26.12)$ \\
\hline No & $1081(65.52)$ \\
\hline N/A & $138(8.36)$ \\
\hline \multicolumn{2}{|l|}{ Importance of Preventive Care } \\
\hline Not Important & $10(0.61)$ \\
\hline Somewhat Important & $167(10.12)$ \\
\hline Important & $578(35.03)$ \\
\hline Very Important & $765(46.36)$ \\
\hline N/A & $130(7.87)$ \\
\hline \multicolumn{2}{|c|}{ Knowledge of Cancer Screenings Needed } \\
\hline Yes & $730(44.24)$ \\
\hline No & $775(46.97)$ \\
\hline N/A & $145(8.79)$ \\
\hline \multicolumn{2}{|c|}{ Up-To-Date on Cancer Screenings } \\
\hline Yes & $269(16.30)$ \\
\hline No & $1091(66.12)$ \\
\hline N/A & $290(17.58)$ \\
\hline
\end{tabular}



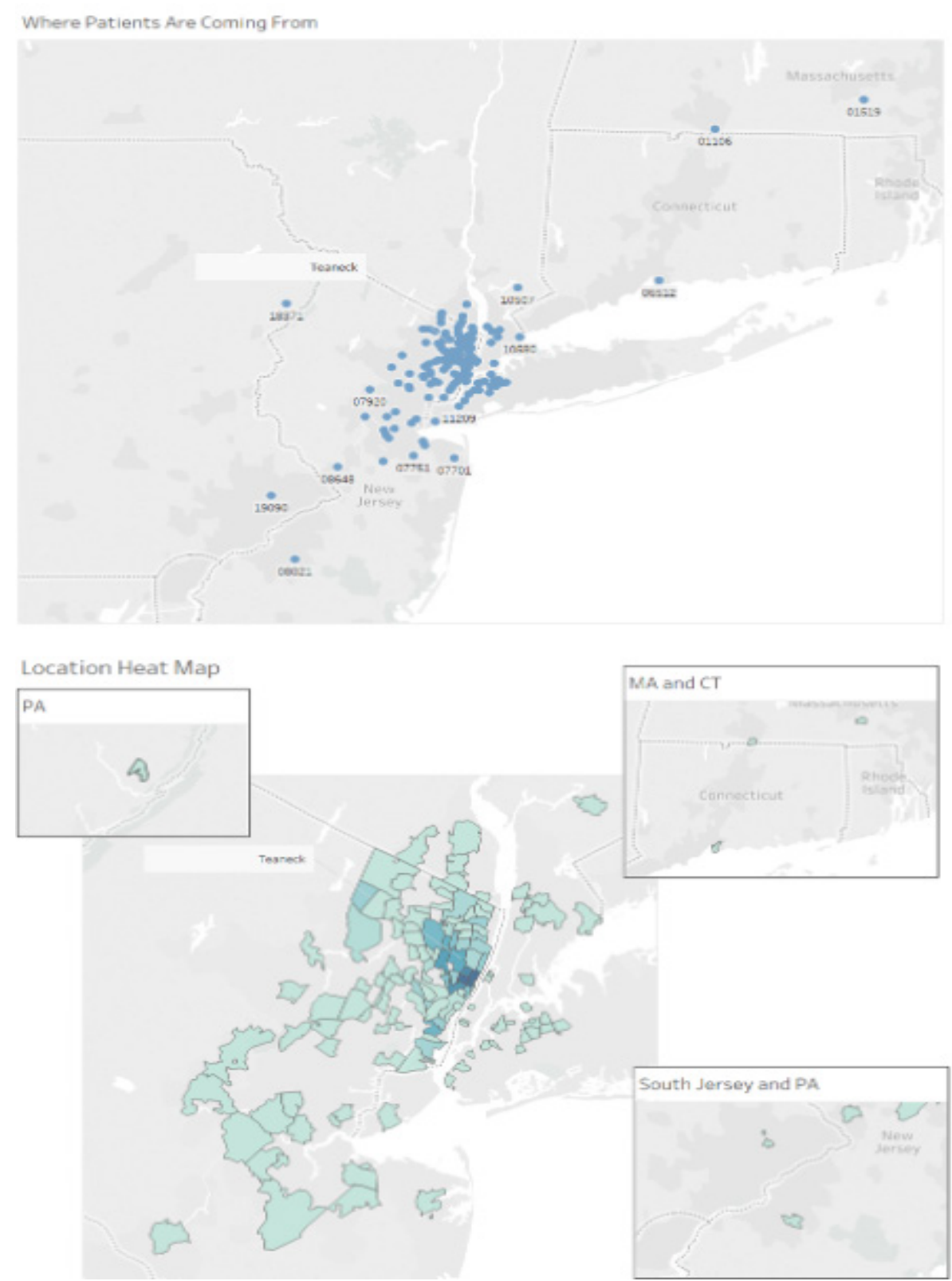

Figure 1. Geographical Distribution of Sample. Distribution in location of origin among survey respondents spanning from South Jersey to Massachusetts.

was the group between 51 and 60 years $(n=533,32 \%)$. Most of the participants had an annual household income of $<\$ 50,000(73 \%)$, with the largest group having an annual household income $<\$ 25,000(n=696,42 \%)$ and $37 \%(n=607)$ of participants received a bachelor's degree. Sociodemographic characteristics are detailed in Table 1.

\section{Preventive Care and Cancer Screenings}

Table 1 shows the responses to questions regarding attitudes and status of medical care and cancer screenings. Regarding access to a primary care physician, $65 \%$ of participants stated that they had a PCP $(n=431)$ while $66 \%(n=1,081)$ reported not having a PCP.

Most participants (92\%) stated that preventive cancer screenings were important, with the largest group saying screenings are "Very Important" ( $\mathrm{n}=765,46 \%)$.

About $44 \%$ of respondents were knowledgeable about the cancer screenings that were needed. Despite these responses, only $16 \%$ of the population responded that they were up-to-date on screening procedures.

In a sample from the 2018 health fair, we found additional data that demonstrated that $72 \%$ of the participants ( $\mathrm{n}=185$ out of 257 ) stated they knew what cancer screenings are recommended but were not up-to-date with cancer screenings (compared to $28 \%$ who knew what cancer screenings are needed and actually were up-to-date on screenings). Meanwhile, $91 \%$ of the participants ( $n=353$ out of 387$)$ who responded that they did not know what cancer screenings were needed stated they were not up-to-date with cancer screenings.

\section{Barriers to Care}

When asked about the barriers to care and preventative health including cancer screenings, the largest barrier to cancer screenings was the lack of insurance $(n=840,40 \%)$. The second largest barrier to care was the cost of seeing 
a physician $(n=517,24 \%)$, followed by not feeling the need to see a physician $(n=299,14 \%)$. Other barriers that were identified included language barrier $(8.0 \%)$, lack of access to care $(5.5 \%)$, fear of seeing a physician $(2.6 \%)$ and lack of transportation (1.4\%).

\section{Discussion}

Despite advances in cancer screening methodologies and treatments, cancer disparities persist among certain racial groups (Hung et al., 2014). In particular, studies have shown that there is a lower cancer screening and survival rate among AAs (Chen, 2005; Bodle et al., 2008; Torre et al., 2016; Jun, 2020). By surveying this population through community health fairs, we found that the majority of AA respondents believed preventive cancer screenings were important $(n=1510,92 \%)$, yet only $16 \%(n=269)$ stated that they were up-to-date on cancer screening procedures.

Many of the barriers that were identified in our questionnaire were also prevalent in other studies in literature, including language barriers, no PCP, lack of insurance and lack of knowledge (Hu, 2008; Hung et al, 2014; Ratushnyak et al., 2019). It is important to identify these barriers because research has shown AAs are diagnosed with cancer at more advanced stages which results in a higher mortality rate and lower survival rate as compared to other racial/ethnic groups (Lin et al., 2002; Jemal et al., 2006).

The largest barrier to cancer screening in our population was a lack of insurance $(n=840,40 \%)$. Further, the majority of respondents noted an annual household income of less than $\$ 49,999$, with the largest group reporting an annual household income of less than $\$ 25,000(n=696,42 \%)$. These findings suggest that the benefit of community health fairs includes providing care to a highly uninsured population and those of low socioeconomic status who may not normally seek care.

Literature supports that the AA population is the least likely racial group to have seen a primary care physician within the last 12 months (Chen, 2015); 65\% ( $=1081)$ of our respondents reported not having a PCP. Therefore, working to increase cancer screening rates through the primary care setting is inadequate for certain Asian communities with a high number of people who do not have, nor visit, a PCP.

Communication difficulties and poor relationships may lead to decreased preventive care maintenance and cancer screenings (Mead et al., 2013). In our survey results, a language barrier was the fourth leading barrier to cancer screenings $(n=169,8 \%)$. Usage of multilingual and culturally competent educational fliers, bilingual healthcare providers and patient navigators may also help lead to increased screening for the AA population.

Some limitations were identified in this study. The data collected from the survey analyzes cross-sectional data. Therefore, we are unable to draw causal conclusions and can only describe results in a non-temporal manner. Our study also did not allow for longitudinal follow-up that would provide insight into the effectiveness of our interventions. Further, we did not have information about respondents' ethnic subgroups. It is important to note that the Asian American population carries heterogeneity within ethnic subgroups, with differences in types of cancer, incidence, and mortality rates dependent on the country of origin. For example, Liver cancer is highest among AA in the Laotian men $(66.1 / 100,000)$, which is 2-4 times higher than Koreans (26), Chinese (21.7), and Filipinos (16.7). Cervical cancer is highest in Cambodian females $(12.7 / 100,000), 3$ times higher than the Chinese (4.5) subgroup (American Cancer Society, 2016). Lastly, we elected to use our own questionnaire, that was influenced and modified from validated surveys to fit the needs and purpose of our health-fairs (Ma et al., 2009).

Despite limitations, this study adds to the evolving literature on cancer screening disparities in AA communities. Implementation of community outreach programs targeted at AAs may attract individuals within the appropriate cancer screening age groups from a large catchment area; specifically, populations in need who lack access to a primary care physician while also linking them to culturally competent care. With our study we examined sociodemographic and screening attitudes as well as identified barriers to screening; providing a framework for actionable steps to improve screenings in the AA communities. Our findings indicate the need for public health promotion, community outreach, and education of the AA community to minimize the gaps in screening rates of this population who continually are impacted by cancer as the leading cause of death.

\section{Author Contribution Statement}

GW and SK contributed to the design and implementation of the research and to the analysis of the results. GW, NA, SK contributed to the writing and revisions of the manuscript. All authors have reviewed the manuscript and approved the final version.

\section{Acknowledgments}

The authors would like to thank the participants in the study. We are grateful to all the public health personnel and healthcare providers who volunteered to assist with the annual health fairs.

\section{Ethical statement}

The study was performed according to the Declaration of Helsinki and approved by the Institutional Review Board of Holy Name Medical Center.

\section{Availability of data}

The datasets generated during and/or analyzed during the current study are not publicly available due to confidentiality but are available from the corresponding author on reasonable request.

\section{Conflict of interest}

The authors declared no potential conflicts of interest with respect to the research, authorship, and/or publication 
of this article.

\section{References}

American Cancer Society (2016). Cancer Facts \& Figures 2016. Accessed April 17, 2021. https://www.cancer.org/research/ cancer-facts-statistics/all-cancer-facts-figures/cancer-factsfigures-2016.html.

Bodle EE, Islam N, Kwon SC, et al (2008). Cancer screening practices of Asian American physicians in New York city. J Immigr Minor Health, 10, 239-46.

CDC Office of Minority Health and Heath Equity (2019). Leading Causes of Death in Males and Females, US. Centers for Disease Control and Prevention. Published November 20, 2019. Accessed April 17, 2021. https://www.cdc.gov/ healthequity/lcod/index.htm.

Chen MS (2005). Cancer health disparities among Asian Americans: what we do and what we need to do. Cancer, 104, 2895-902.

Torre LA, Sauer AMG, Chen MS, et al (2016). Cancer statistics for Asian Americans, Native Hawaiians, and Pacific Islanders, 2016: Converging incidence in males and females. CA Cancer J Clin, 66, 182-202.

Hastings KG, Jose PO, Kapphahn KI, et al (2015). Leading Causes of Death among Asian American Subgroups (2003-2011). PLoS One, 10, doi:10.1371/journal.pone.0124341.

Hu K-Q (2008). Hepatitis B virus (HBV) infection in Asian and Pacific Islander Americans (APIAs): how can we do better for this special population?. Am J Gastroenterol, 103, 1824-33.

Hung M-C, Liu M-T, Cheng Y-M, Wang J-D (2014). Estimation of savings of life-years and cost from early detection of cervical cancer: a follow-up study using nationwide databases for the period 2002-2009. BMC Cancer, 14, 505.

Jemal A, Siegel R, Ward E, et al (2006). Cancer statistics, 2006. CA Cancer J Clin, 56, 106-30.

Jun J (2020). Cancer/health communication and breast/cervical cancer screening among Asian Americans and five Asian ethnic groups. Ethn Health, 25, 960-81.

Lin SS, Clarke CA, Prehn AW, et al (2002). Survival differences among Asian subpopulations in the United States after prostate, colorectal, breast, and cervical carcinomas. Cancer, 94, 1175-82.

Ma GX, Shive SE, Wang MQ, Tan Y (2009). Cancer screening behaviors and barriers in Asian Americans. Am J Health Behav, 33, 650-60.

Ma GX, Toubbeh JI, Wang MQ, et al (2009). Factors associated with cervical cancer screening compliance and noncompliance among Chinese, Korean, Vietnamese, and Cambodian women. J Natl Med Assoc, 101, 541-51.

Mead EL, Doorenbos AZ, Javid SH, et al (2013). Shared decision-making for cancer care among racial and ethnic minorities: a systematic review. Am J Public Health, 103, e15-29.

Ratushnyak S, Hoogendoorn M, van Baal PHM (2019). Costeffectiveness of cancer screening: Health and Costs in Life Years Gained. Am J Prev Med, 57, 792-9.

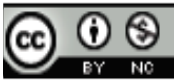

This work is licensed under a Creative Commons AttributionNon Commercial 4.0 International License. 